\title{
Relation between benchmark displacement velocity and seismic activity caused by underground longwall exploitation
}

\author{
Józef Duda ${ }^{1}$
}

Received: 20 July 2015/Accepted: 1 December 2015/Published online: 18 December 2015

(C) The Author(s) 2015. This article is published with open access at Springerlink.com

\begin{abstract}
The following paper addresses the problem of mining-induced surface subsidence by giving consideration to the aspect of displacement velocity and its relation to seismic activities recorded in the area of benchmarks. A series of geodetic measurements were carried out in order to develop an accurate description of the deformation process. The mathematical model of a benchmark displacement process was worked out to estimate the maximum deformation velocity and the time of maximum velocity occurrence. The comparison between the measured and calculated data was presented. The displacement velocity value was compared to the seismic activity recorded during the exploitation period. Four cases were thoroughly investigated as they represent types of relation between the seismic activity and the deformation velocity.
\end{abstract}

Keywords Mining surface deformation - Displacement velocity · Induced seismic activity

\section{Introduction}

Mining-induced surface deformation and mine tremors are the negative consequences of mining activities that affect the safety and condition of buildings, roads and other engineering objects on the surface. A widely used method for surface subsidence prediction is the influence function method that is applied to predict such surface deformation parameters as subsidence, horizontal displacement, slope, curvature and horizontal strain (e.g. Cai et al. 2014; Deck and Anirudh 2010; Saeidi et al. 2009; Knothe 2005). The use of GNSS/GPS technology is playing an increasing role in the surface deformation monitoring of mining areas (e.g. Bian et al. 2014; Doležalová et al. 2009).

Józef Duda

jo.duda@po.opole.pl

1 Opole University of Technology, Opole, Poland 
Mining-induced seismicity is characterized by two types of seismic events. The first one is induced directly by the seismic activity and it is related to rockmass fracturing in front of a longwall face and is dependent on the exploitation method and its rate. The second type is allied with the tectonic discontinuities motion and it is characterized by higher energy values and the location of foci is independent on the setting of a temporary mining front. Both types are influenced by local geological conditions (Gibowicz and Kijko 1994).

The possibility of providing a massive tremor forecast, on the base of cyclic geodetic observations of a subsidence trough, together with the observation of the total seismic energy released during the exploitation, was analyzed by Sokoła-Szewioła (2009). Their research results confirmed the relation between the seismic activity and the surface deformation velocity and were depicted by Bańka (2000) on the base of regular geodetic measurements carried out on the surface of the subsidence trough, together with seismic observations. The seismic activity pinpointed in both mentioned elaborations is covered by the notion of two indices: the number of seismic events $N$ and the energy released by tremors observed during the exploitation $E$, recorded within the time unit. Sokoła-Szewioła (2009) developed the algorithm which enabled to forecast tremors of energy above $5 \times 10^{4} \mathrm{~J}$. Ex post normalized, the mean square error of a forecast against a real occurrence of seismic events was calculated at 0.12 , and the surface subsidence velocity was proved to be related to the number of seismic events recorded during the exploitation. The method applied in the alluded elaboration derived from the observations of seismic events recorded over the entire excavation area and the obtained seismic records included 339 events of the energy value above $10^{3} \mathrm{~J}$, while the prediction algorithm concerned 216 events of energy value above $5 \times 10^{4} \mathrm{~J}$ (Sokoła-Szewioła 2009). The released energy and the number of events correlate, thus the number of seismic events is the efficient parameter for such an analysis.

These analyses and observations lead to the assumption that a subsidence trough formation, represented by the surface deformation velocity, is partly dependent on the seismic activity level, represented by the number of seismic events observed within the time unit.
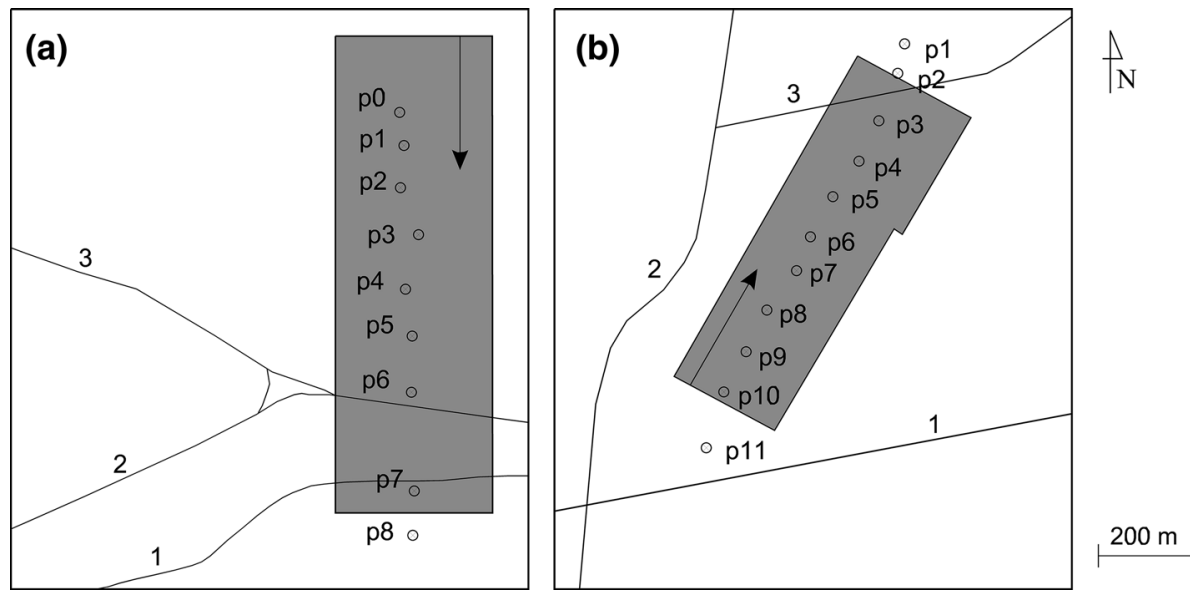

Fig. 1 Localization of benchmarks in the area of the excavation, a longwall 3/503 in "Bobrek-Centrum" area, b longwall 183/418 in "Pokój" area. The grey field indicates the exploitation area, p0-p11benchmarks, 1-railways, 2, 3-important roads, black arrow marks the direction of excavation works 
The purpose of the current research is to probe the question about the conditions required to describe the relation between the number of seismic events and the deformation velocity in the area of underground mining activity. To achieve this goal, cyclic geodetic measurements were conducted on the surface of the subsidence trough. The results were compared to seismic records in order to describe the relation between these two processes.

In the course of the study, the formula was proposed to describe the transient displacement vector value as a function of time and, as such, it was applied to calculate the displacement velocity maximum value and the event time. Additionally, the GPS technique of surface geodetic monitoring was implemented to obtain the displacement vector value instead of the subsidence value. The new approach, concerning the relation between the displacement velocity observed on the surface jointly with the number of seismic events recorded in the area, imposed a limitation on the number of seismic events according to the appropriately small distances of the epicentres to the benchmarks.

\section{Methodology and area of research}

The presented tests were carried out in two mining areas. The first was the longwall 3/503 mining area in "Bobrek-Centrum", situated in the city of Bytom, Poland (Fig. 1a) and the remaining measurements were taken on nine bench marks $\mathrm{p} 0-\mathrm{p} 8$ localized along the longer axis of the exploitation area. Geodetic estimations were obtained in 21 cycles launching on 17.04.2009 and completed on 22.10.2010. The seismic record contains data from 14.04.2009 till 24.06.2010. The period 14-28.04.2009 involved preparation works while the exploitation itself was initiated on 29.04.2009 and lasted till 24.06.2010. All in all, the measurements had launched several days before the exploitation began and they lasted for 4 months after its completion on order to acquire residual displacement figures. The longwall mining area 183/418 in "Pokój" is situated in the city of Ruda Śląska, (Fig. 1b) several kilometres away from the "Bobrek-Centrum" area. The geodetic record was compiled on eleven benchmarks p1-p11 situated along the longer axis of the exploitation area. 15 evaluation series were administered, from 14.08.2007 till 8.05.2009. The seismic record contains data, from 21.05.2007 till 24.10.2008. This time, the assessments launched 3 months after the exploitation had begun and they lasted for 7 months after its completion. As seismic observation in both cases had started before geodetic evaluations launched, it was the beginning of seismic observation that marked the starting point for further calculations, as demonstrated on the figures (from 2 to 5). In both areas, the exploitation was performed at the depth of about $700 \mathrm{~m}$ below the ground level.

The GPS technology was applied to the geodetic measurements which were taken with the use of a static method, with a reference to Active Geodetic Network EUPOS stations, localized several kilometres away from the research areas. Post processing works were carried out to calculate spatial coordinates of each benchmark with the accuracy between 1 and $52 \mathrm{~mm}$. The application of the GPS technology to cyclic measurements enables to gain displacement vector components $r_{x}, r_{y}$ and $r_{z}(u, v$ and $w)$. On the base of these components one can calculate the displacement vector value $r_{1}$, as the function of time. Since the displacement vector value is a geometrical sum of three components, its accuracy is between 7 and $68 \mathrm{~mm}$. The displacement velocity value can be calculated as the displacement value increment is divided by time between two series. The velocity value is affected by a measurement error, ranging $0.1-2.8 \mathrm{~mm} /$ day. Major velocity errors stem 
from the GPS method accuracy that is relatively low in comparison to optical methods like precise nivelation.

The current state of art suggests the accuracy of the applied GPS method of about 5-20 mm for a spatial point position (e.g., Doležalová et al. 2009). The accuracy of optical methods like precise nivelation, applied by Sokoła-Szewioła (2009) and Kowalski (2004) is about $1 \mathrm{~mm}$. However, it enables to achieve only the vertical component of the deformation, i.e. the subsidence, without any further information concerning horizontal components of such a displacement. The horizontal displacement plays a profound role in the deformation process (e.g., Cai et al. 2014).

As the data on seismic events derives from the seismic network administered by mines, tremors of energy equal or higher than $1 \mathrm{~kJ}$ are critical for the seismic recording. Tremors of lower energy magnitude were observed in the area but they were not recorded since the number of stations did not allow to obtain complete record of tremors with the energy value below this limit. Such a limitation of seismic records was applied in former researches, too (e.g., Sokoła-Szewioła 2009). Seismic records from both areas contain 2281 events, while 1553 events of energy between $1 \mathrm{~kJ}$ and $800 \mathrm{MJ}$ were observed in "BobrekCentrum" and 728 events of energy ranging 1 kJ-6 MJ were observed in "Pokój".

Moreover, seismic events with their epicentres situated no further than $290 \mathrm{~m}$ at maximum from the benchmark in "Bobrek-Centrum" area and $280 \mathrm{~m}$ at maximum in "Pokój" area, were analyzed. The distance of $280-290 \mathrm{~m}$ is the radius of the main influences deriving from the influence function method. This method is widely applied to forecast surface deformation parameters (e.g. Cai et al. 2014; Knothe 2005). The radius of the main influences depends on the exploitation depth and the angle of main influences that results from local geological and mining conditions. Since most tremors are assumed to be mining-induced ones and are localized above the exploited seam, the radius of the main influences is assumed to be the maximum horizontal distance of the tremor focus that can affect the surface deformation velocity value.

\section{Modelling of displacement velocity}

The model function of displacement as a function of time was proposed by Knothe (1984), first postulated in the fifties, and it is still applied to solve numerous problems such as an estimation of time allocated for the surface deformations occurrence instigated by the exploitation works beneath. (e.g., Knothe 2005; Kowalski 2001). The basic form of a time function is described by the Eq. (1):

$$
w(t)=w_{k}\left|1-e^{-c\left(t-t_{0}\right)}\right|,
$$

where $w(t)$ is the function describing the subsidence process; $t$ denotes the time, $w_{k}$ is the final (maximum) subsidence, $c$ is the coefficient characterizing the rate of transition influence through the rock mass, related to local geological conditions and the exploitation intensity in the past, $t_{0}$ is the time of exploitation front passing under the benchmark.

As the function (1) describes the subsidence process since from the moment of maximum subsidence velocity, the modification (2) was introduced (Kochmański 1980):

$$
w(t)=\sum_{i=1}^{i=n} w_{k_{i}}\left|1-e^{-c\left(t-t_{i}\right)}\right|,
$$


where $w_{k i}$, are the partial subsidence steps, $t_{i}$ are the time steps; $n$ is the number of steps, generally $<10$; the argument $t$ and the coefficient $c$ are compatible with (1) the formula. This modification improved the goodness of fit of the model function to the measured data but it caused multiplication of the number of parameters, what made the function difficult to apply.

It was assumed that the theoretical subsidence is a sum of partial subsidence's, caused by extracting subsequent rectangular blocks (Kowalski 2001). The subsidence value in time is then expressed by the formula (3), (Kowalski 2007):

$$
w(t)=C \int_{\tau=0}^{\tau=t} q\left(t^{\prime}-\tau\right) \cdot T(\tau) d \tau
$$

where

$$
q\left(t^{\prime}-\tau\right)=g\left(t^{\prime}-\tau\right) v_{f}\left(t^{\prime}-\tau\right) \exp \left[-\alpha \cdot x^{2}\left(t^{\prime}-\tau\right)\right]
$$

and

$$
T(\tau)=\left\{\begin{array}{ccc}
0 & \text { for } & 0 \leq \tau \leq \tau_{0} \\
1-A \exp \left[-c\left(\tau-\tau_{0}\right)\right] & \text { for } & \tau>\tau_{0}
\end{array}\right.
$$

where $C$ is the parameter related to geological and mining conditions and denotes the maximum forecasted subsidence to seam thickness ratio, $\alpha$ is the coefficient dependent on the radius of the main influences, $g\left(t^{\prime}-\tau\right)$ is the seam thickness, $v_{f}\left(t^{\prime}-\tau\right)$ is the exploitation front velocity, $x\left(t^{\prime}-\tau\right)$ is the horizontal distance between the benchmark and the exploitation front, $t^{\prime}$ is the time of the exploitation front passing under the benchmark. Parameters $C, g, v_{f}$ and $\alpha$ are the a priori data, based on geological and mining conditions. The argument $t$ and parameter $c$ are compatible with (1) the formula, $A$ is a coefficient (with value between 0 and 1 ), $\tau_{0}$ is the time lag. $A, c$ and $\tau_{0}$ are a posteriori parameters, determined using the least squares method.

If one assumes $A$ parameter as 1 , then the function (3b) equals the time function (1). The factor $\exp \left[-\alpha^{\prime} x^{2}\left(t^{\prime}-\tau\right)\right]$, from (3a) the formula is Gaussian distribution used in the influences function method to forecast the parameters of surface deformation (e.g., Cai et al. 2014; Knothe 2005; Kowalski 2004; Bańka 2000; Knothe 1959). The derivative of (3) the function in respect to time is the function of subsidence velocity in time and its shape resembles the Gaussian distribution. The model (3) can be further developed (Kowalski 2007) by adding a parameter to (3b) the function in order to improve the fit of the model to the experimental data.

The application of different function types to describe the deformation process was proposed by Bogusz and Mendecki (2011) to find the time of the maximum subsidence velocity and, subsequently, to compare it with the position of a longwall front and the seismic activity (4):

$$
h(t)=a_{1}\left[1-\left(\exp \left\{\frac{t-a_{2}}{a_{3}}\right\}+1\right)^{-1}\right],
$$

where $a_{1}$ is the maximum observed subsidence, $a_{2}$ is the time of the maximum velocity occurrence, $a_{3}$ is the parameter describing the function curvature. Parameter $a_{3}$ can be related to an inversed $c$ parameter from (1), (2) and (3) formulas. Parameters $a_{1}, a_{2}$ and $a_{3}$, are then estimated a posteriori on the base of the least squares method to gain the best fit of 
calculated to measured data. The function (4)'s derivative with respect to time, describes the subsidence velocity and is characterized by one extremum that is related to the time of the most intensive surface deformation.

The function (3) requires four a priori parameters whereas three a posteriori ones are obtained with the use of the least squares method to gain the best fit to the observed data. The function (4) has three parameters, estimated a posteriori on the base of the least square method, and it requires no a priori parameters.

In order to estimate the deformation velocity value during the exploitation period and to reduce the number of a posteriori parameters, the formula (5) was proposed. As the geodetic measurements were recorded in periods of time with irregular intervals, the aim of the function was to interpolate the displacement vector value in each time unit.

$$
r_{\text {calc }}(t)=d \cdot \sum_{\tau=0}^{\tau=t} e^{-b} \frac{b^{\tau}}{\tau !},
$$

where $r_{c a l c}$ is the calculated displacement, $b$ is the model parameter that can be interpreted as the time of local velocity maximum occurrence, $d$ is interpreted as the final displacement, $t$ denotes the time. The aim of $r_{\text {calc }}$ function is to generate the best fit to the measured displacement function $r_{\text {meas }}$, on the base of the following condition (6):

$$
R_{1}=1-\frac{\sum_{t_{\min }}^{t_{\max }}\left|r_{\text {meas }}(t)-r_{\text {calc }}(t)\right|}{\sum_{t_{\min }}^{t_{\max }} r_{\text {meas }}(t)},
$$

where $t_{\min }$ and $t_{\max }$ are the times of the first and the last displacement measurements.

The parameters were accepted when the function $R_{1}$ reached its maximum value. The coefficient $R_{1}$ was based on L1 norm that is resistant to high, single deviations of the measured values (e.g. Dębski 2010), and was used as a tool to solve such seismological problems as an approximation of the parameters of frequency - the magnitude distribution (FMD) of earthquakes (Wiemer and Wyss 2002). However, the application of the least squares method gave comparable results (Table 1).

The parameter $d$ value is generally lower than the maximum $r_{\text {meas }}$ value, what is caused by residual displacements, observed longitudinally after the completion of the exploitation. The parameter $d$ is then unconstrained, thus the function $r_{2}$ involves two independent parameters $d$ and $b$. Solving a problem of finding a maximum of (5) the function is forward and unique in character, and it is possible to apply it by any program for matrices calculations.

Table 1 Values of $R l$ coefficients and parameters $b$ and $d$, calculated with the use of (6) formula, for cases presented in Figs. 4a, b and 5a, b

\begin{tabular}{lllll}
\hline Calculated quantity & $4 \mathrm{a}$ & $4 \mathrm{~b}$ & $5 \mathrm{a}$ & $5 \mathrm{~b}$ \\
\hline$R 1$ & 0961 & 0930 & 0964 & 0861 \\
$b$ (week number) & $45(45)$ & $55(55)$ & $21(22)$ & $35(36)$ \\
$d(\mathrm{~cm})$ & $135(137)$ & $38(38)$ & $229(227)$ & $124(125)$ \\
\hline
\end{tabular}

The $b$ and $d$ parameters presented in brackets are calculated with the use of the least squares method 

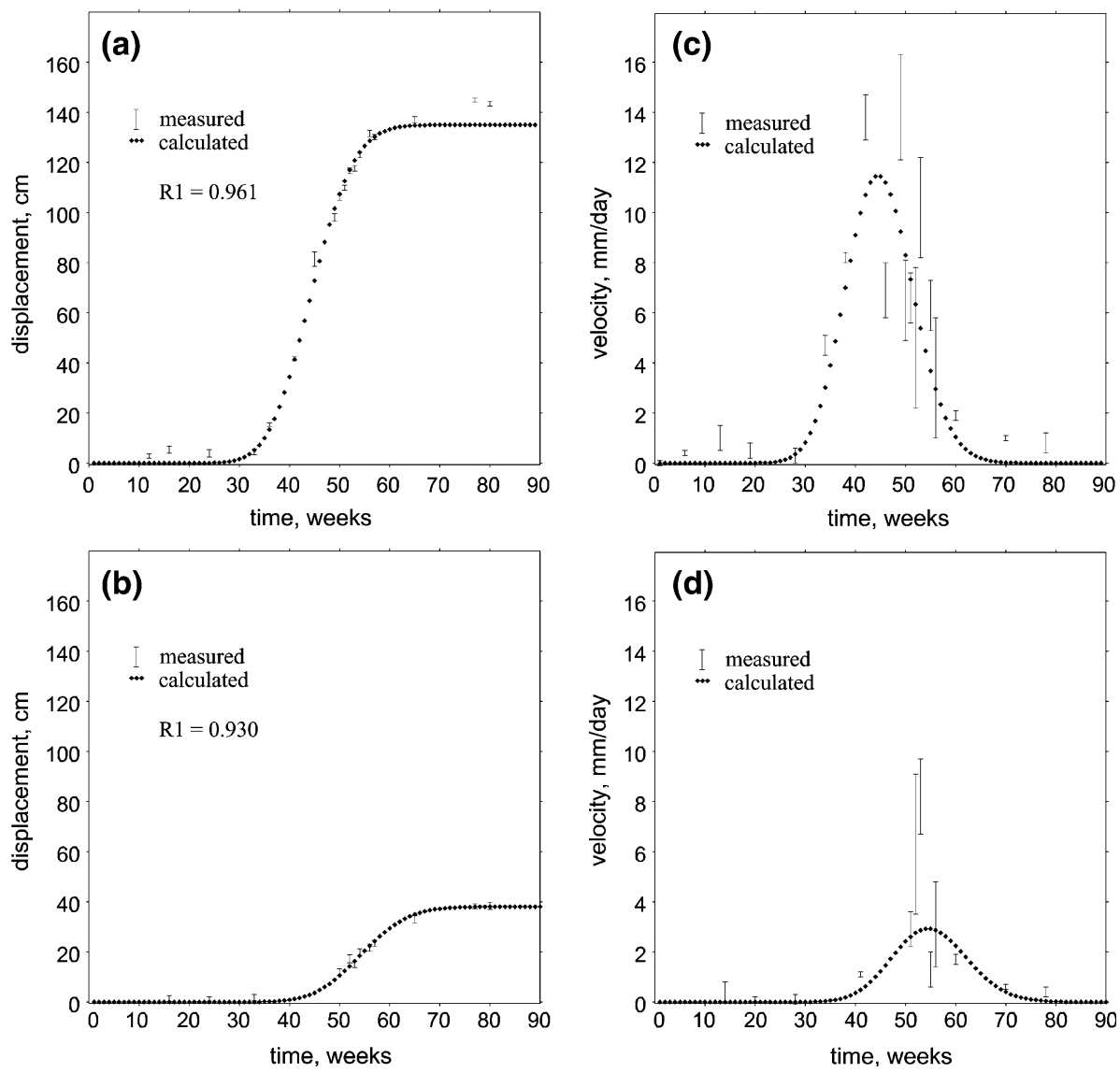

Fig. 2 Comparison of the calculated data versus the measured data for the displacement vector value (left) and displacement velocity (right), for: a, c benchmark p5 in "Bobrek-Centrum" area; $\mathbf{b}$ and $\mathbf{d}$ benchmark p8 in "Bobrek-Centrum" area

The proposed model function of displacement (5) was compared to the measured values (Figs. 2a, b, 3a, b). The function (5) is discrete and cumulative in character, therefore the differentiation quotient of this function represents the calculated velocity $v_{\text {calc }}(7)$ :

$$
v_{\text {calc }}(t)=d \cdot e^{-b} \cdot \frac{b^{t}}{t !},
$$

where the parameters are compatible with the (5) formula.

The comparison of velocity, calculated on the base of the model function (7), and the measured velocity value is presented in Figs. $2 \mathrm{c}, \mathrm{d}$ and $3 \mathrm{c}$, d. The velocity function is characterized by one maximum.

Generally, the calculated velocity is underestimated in comparison to the data measured in the cases presented in Fig. 2, and it is overestimated in the cases presented in Fig. 3. The calculated velocity value is characterized by a high relative error. The goodness of fit is illustrated by coefficients $R_{1}$ (formula 6), which are presented in the Figs. 2 and 3 and 

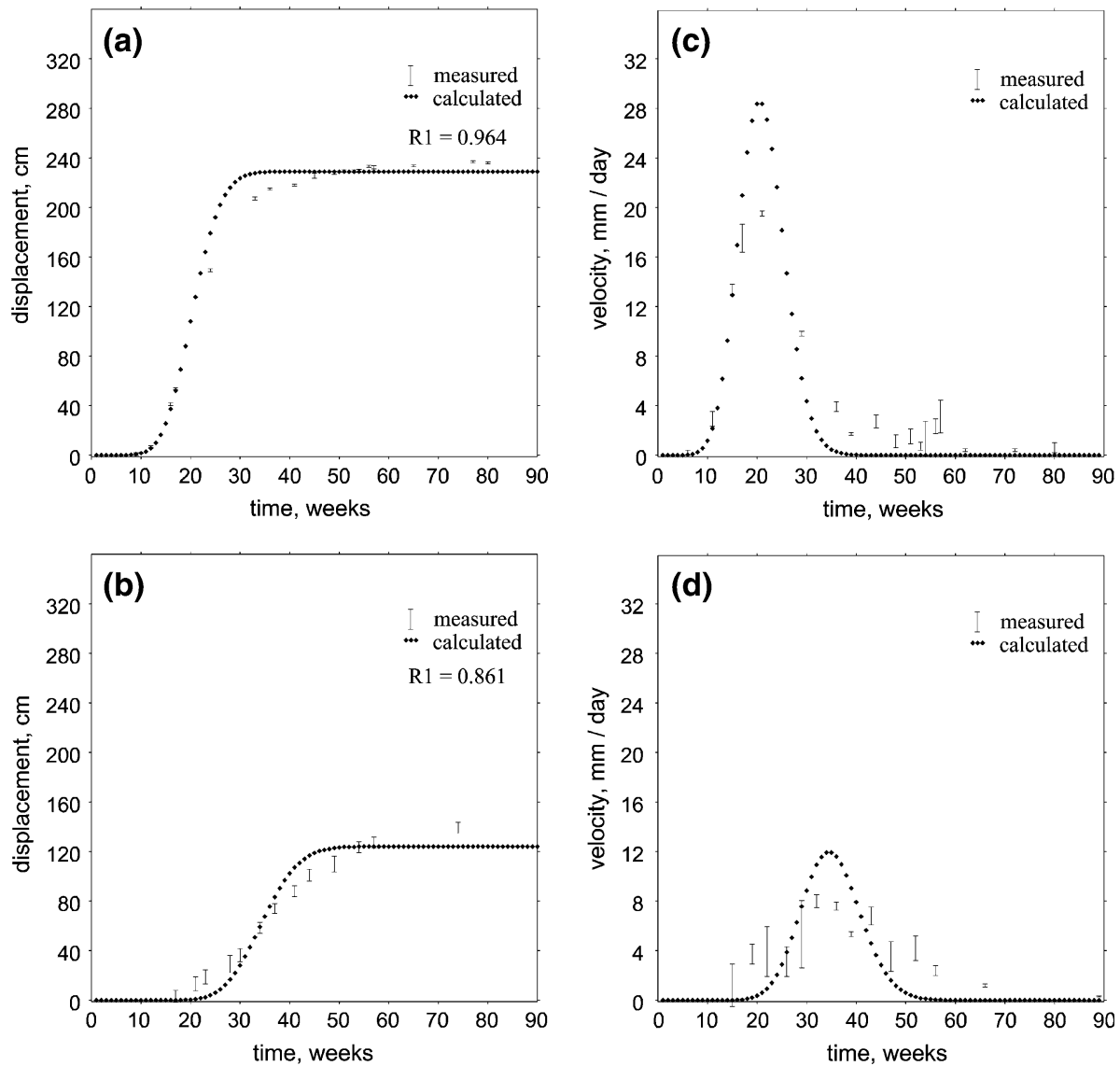

Fig. 3 Comparison of the calculated data versus the measured data for the displacement vector value (left) and displacement velocity (right), for: $\mathbf{a}$, $\mathbf{c}$ benchmark p0 in "Bobrek-Centrum" area; $\mathbf{b}$ and $\mathbf{d}$ benchmark p8 in "Pokój" area

listed in Table 1 . The coefficient $R_{1}$ depends on a relative difference between the measured and calculated data.

\section{Results}

The calculated velocity ( $v_{\text {calc }}$-formula 7 ) was compared to the seismic activity that was represented by the number of seismic events reported within the time unit. Figure 4 presents the comparison between the calculated velocity versus the seismic activity for two benchmarks p5 and p8 localized in "Bobrek-Centrum" area (Fig. 4a, b respectively) whereas Fig. 5 presents this comparison for the benchmark p0 in "Bobrek-Centrum" area and the benchmark p8 localized in "Pokój" area (Fig. 5a, b respectively). These diagrams illustrate four different situations - corresponding to the relation types between the seismic activity and the deformation velocity. 

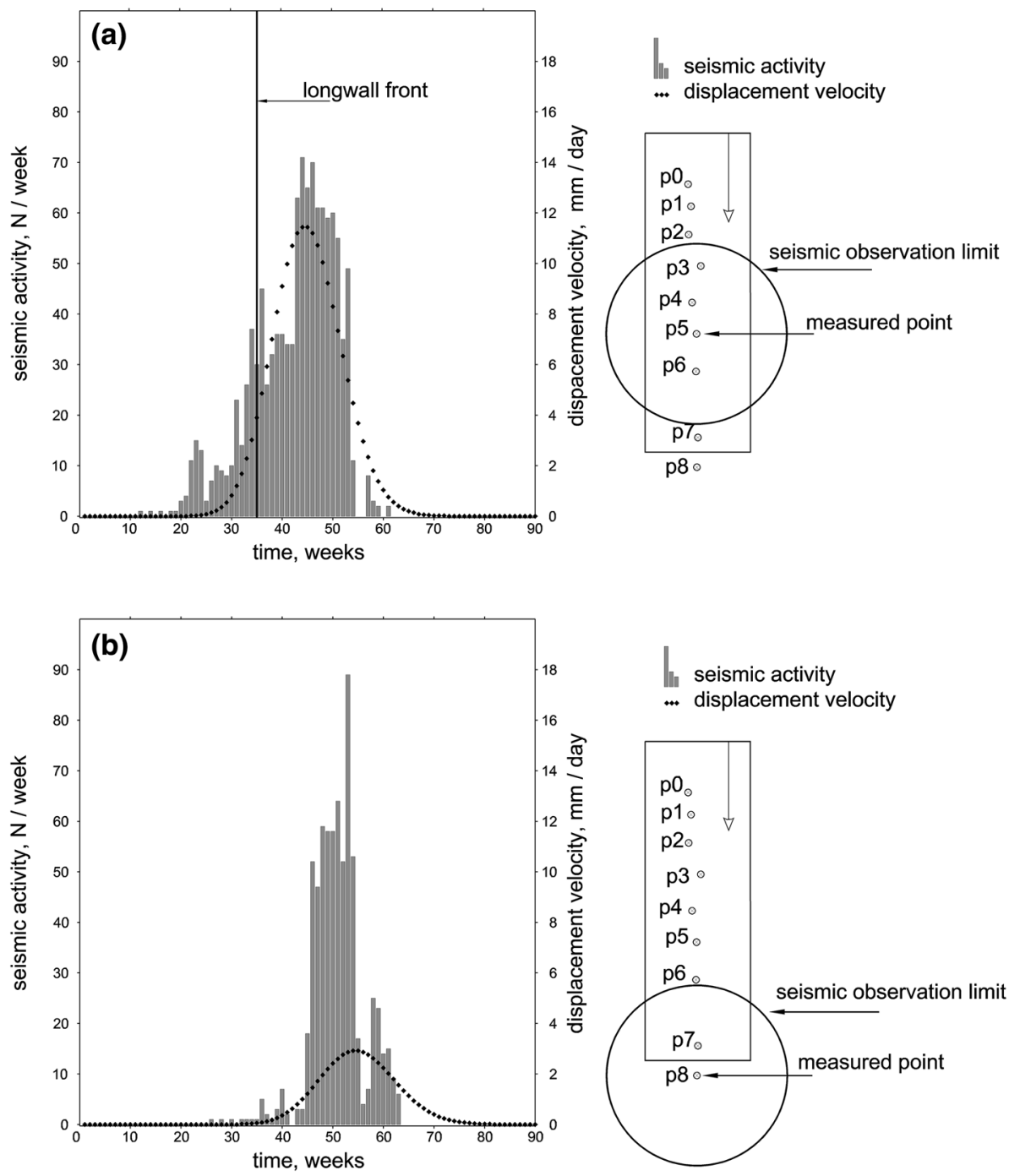

Fig. 4 Comparison of the seismic activity versus displacement velocity, a for benchmark p5, and b benchmark p8 in "Bobrek-Centrum" area

Figure 4 represents the case of the distinct maximum of the seismic activity, accompanied by the maximum of the displacement velocity. In Fig. 4a, both the maximum displacement velocity and the maximum seismic activity are delayed according to the time of the exploitation front passing under the benchmark. In Fig. $4 b$, the exploitation front does not pass under the benchmark p8 because it is situated outside the exploitation area. Both cases are characterized by the relatively high seismic activity accompanied by the medium and low displacement velocity (Table 2). Both presented cases in Fig. 4, represent a qualitative relation between the displacement velocity and the seismic activity observed over the area, limited by the radius of the main influences, (represented by the circles on 

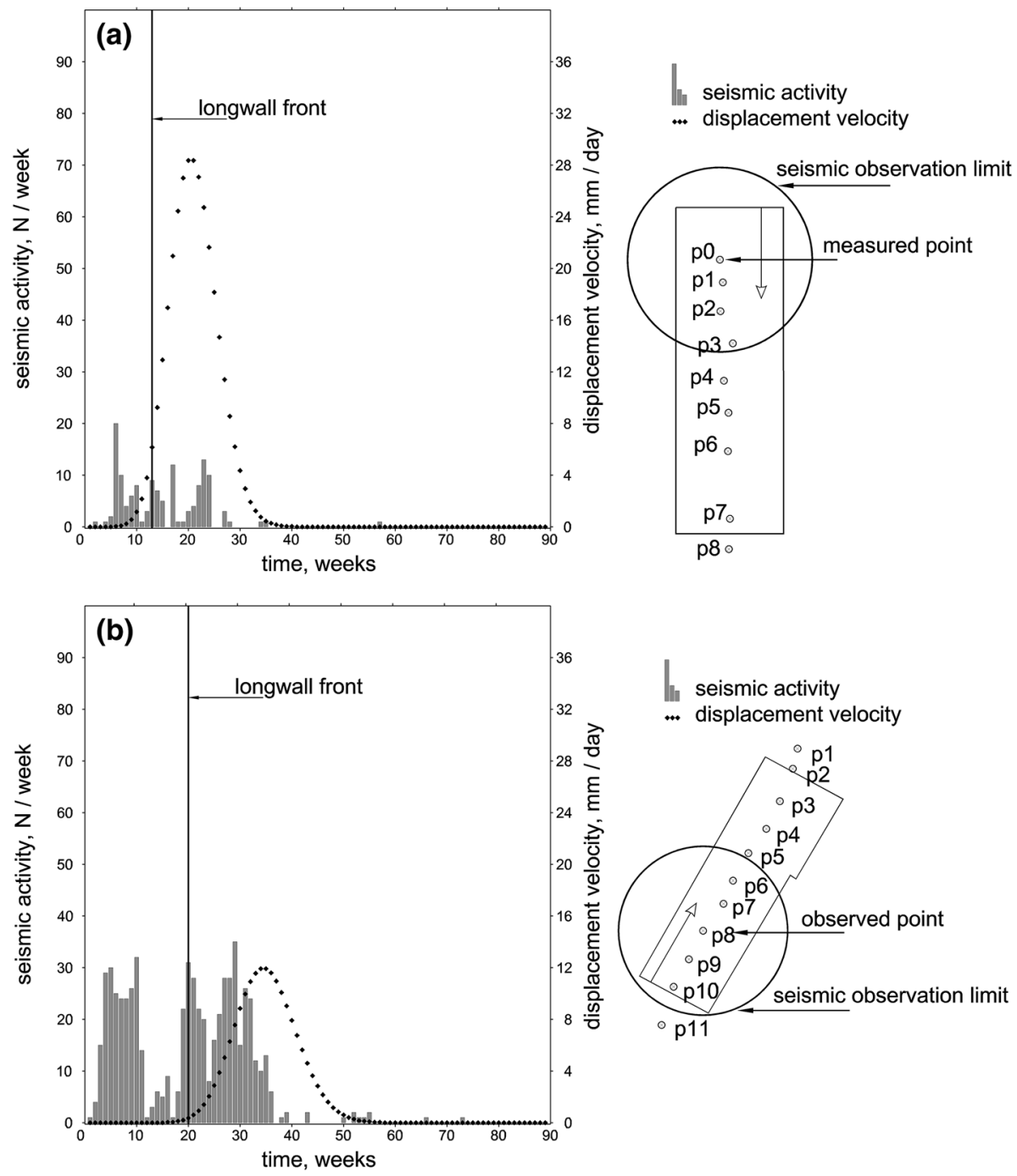

Fig. 5 Comparison of the seismic activity versus displacement velocity, for a benchmark p0 in "BobrekCentrum" area, and b benchmark p8 in "Pokój" area

the insets) (Fig. 4). The displacement velocity maximum follows the seismic activity maximum.

Figure 5 represents the two cases where the seismic activity is not characterized by one distinct maximum. In both cases the maximum displacement velocity is delayed according to the time of the exploitation front passing under the benchmark. The case presented in Fig. 5a is characterized by the very high displacement velocity, up to $30 \mathrm{~mm} /$ day and the relatively low seismic activity, while the case in Fig. 5b represents a situation where both values of the seismic activity and the displacement velocity are at the medial levels (Table 2). The cases presented in Fig. 5 pinpoint either a minor relation or lack of a qualitative relation between the values under question observed in the area limited by the 
Table 2 Values of total numbers of seismic events $N$, maximum seismic activities $N /$ week and maximum calculated velocities $v_{\text {calc }}$, for cases presented in Figs. $4 \mathrm{a}, \mathrm{b}$ and $5 \mathrm{a}, \mathrm{b}$

\begin{tabular}{lllll}
\hline Quantity & $4 \mathrm{a}$ & $4 \mathrm{~b}$ & $5 \mathrm{a}$ & $5 \mathrm{~b}$ \\
\hline Total $N$ & 1146 & 703 & 137 & 636 \\
Max $N /$ week & 71 & 89 & 20 & 35 \\
Max $v_{\text {calc }}(\mathrm{mm} /$ day $)$ & 11.5 & 2.9 & 28.4 & 11.9 \\
\hline
\end{tabular}

radius of the main influences, (represented by circles on the insets) (Fig. 5). The displacement velocity is independent of the seismic activity.

Table 1 represents the parameters of (5) the function applied to calculate the displacement vector value as well as the displacement velocity and the coefficients of goodness of fit calculated with the use of (6) the formula. The cases represented by Figs. 4a and $5 \mathrm{a}$ prove to achieve the highest goodness of fit whereas those in Fig. 5b manifest the lowest goodness of fit.

Table 2 represents the most important parameters of the observed seismic activity and the calculated displacement velocity value. It is important to highlight the highest displacement velocity observed in the case of the lowest seismic activity (Fig. 5a) while the highest seismic activity is accompanied by the medium value of the displacement velocity (Fig. 4a).

Both medium and high seismic activities, characterized by one maximum (Fig. 4a, b) can be related to the displacement velocity process where the maximum of velocity is accordant to the maximum of the seismic activity. On the other hand, medium and low seismic activities, not characterized by one distinct maximum (Fig. 5a, b), lack any connection with the displacement velocity calculated with the use of (7) the formula. The maximum displacement velocity is observed with the delay in relation to the exploitation front passing under the benchmark (Figs. 4a, 5a, b). The benchmark localized outside the exploitation area (Fig. 4b) is characterized by the low displacement velocity.

\section{Discussion}

The process of a trough subsidence is a complex phenomenon characterized by the subsidence velocity influenced by many factors, such as breaks in the course of the exploitation. The issue is investigated by Kowalski (2004) on the base of the geodetic measurements conducted with a daily frequency with the focus on the transient surface deformation. The results presented by this author prove the occurrence of numerous local maxima of the subsidence velocity what leads to the assumption that the velocity fluctuations can result from breaks in the exploitation process. In this view, Bańka (2000) and Sokoła-Szewioła (2009) analyze the deformation velocity fluctuations and relate them to the changes of the seismic activity. The following research juxtaposes the seismic activity with the displacement velocity under various seismic conditions and investigates thoroughly the deformation process observed from the initial stage to the residual one (Sect. 2).

The proposed model function $r_{\text {calc }}$, described by the formula (5) bases on the notion assuming the existence of one maximum of the deformation process intensity, similarly to (1) and (4) models. The application of the (3) model results in the identification of either one maximum of the subsidence velocity with the speculation of a constant front advance velocity $v_{f}$, or numerous local maxima of the subsidence velocity including weekend 
breaks in the course of the exploitation (e.g. Sroka 2009; Kowalski 2004). The main significance of application function (3) is the improvement of the forecast of the mininginduced subsidence with the aspect of engineering impact on the objects on the surface. The (5) function, proposed by the author, is devoted to establish the time of the maximum deformation velocity value in order to compare it with the seismic activity and the longwall position. The displacement function (5) has two parameters, best fit is achieved either by finding the maximum of (6) the function or with the use of the least squares method with comparable results. Generating the best fit for a two-parameter function is unique in case of the experimental data, while for a three-parameter function it depends on the initial parameters. That allows the estimation process to become automatic in case of the (5) function parameters. The displacement function (5) can be applied to different data measurement sets with the measurement data only, while the application of the (3) function requires detailed knowledge of mining and geological conditions.

The main disadvantage of the proposed model (Eqs. 5 and 7) is lack of continuity of the function, which leads to the need of estimation a time step. In this case, the time step is 1 week ( 7 days). That assumption causes difficulties in comparing current results to the results obtained from the former models. Another disadvantage concerns the parameter controlling the function slope, like the $c$ parameter in (1), (2) and (3) models and the $a_{3}^{-1}$ parameter in the (4) model. The parameter controlling the function slope can be related to the geological conditions and rockmass fracturing caused by former exploitation. Lack of this parameter makes it difficult to compare the presented results to the former ones in terms of rockmass vulnerability to the underground exploitation. Nevertheless, the calculated displacement velocities are comparable to the values gained with the use of the daily geodetic measurements, presented in the literature (e.g. Kowalski 2004), where the subsidence velocity reached $50 \mathrm{~mm} /$ day. The calculated velocities that reached $30 \mathrm{~mm} /$ day are comparable and to the measured values (Figs. 2 and 3).

The relation between the seismic activity and the benchmark displacement velocity can be observed only in case of the high seismic activity generated by the exploitation. This relation can be observed also outside the exploitation area in case of a very low displacement velocity accompanied by high seismic activity (Fig. 4b). Nonetheless, in case of low seismic activity, this relation between seismic activity and benchmark displacement velocity is not observed in the following research.

\section{Conclusions}

This paper describes the novel method for measurement of the relation between the displacement velocity observed on the surface of a mining area and the seismic activity induced by the excavation process. In the course of the studies, GPS geodetic measurements were carried out with the frequency of one to several weeks which allowed for the displacement vector value estimation. The application of the displacement function (5) enabled to gain the deformation velocity as a function of time and, therefore, to estimate the time of the maximum process intensity. The comparison of the benchmark displacement velocity and the number of seismic events with epicentres situated within the distance of the radius of the main influences, and with the seismic energy value above $1 \mathrm{~kJ}$, manifested a qualitative relation in a limited number of observations.

The methodology applied for the sake of this study to the two mining areas with the longwall exploitation system exposed two major issues of importance. Firstly, the relation 
between the displacement velocity and the seismic activity was possible to be observed in case of the high seismic activity where the total number of seismic events was above 1000 , the maximum activity was above 50 events per week (Fig. 3). Secondly, the relation between the displacement velocity and the seismic activity was not possible to be observed in case of the low seismic activity, where the total number of seismic events was between 100 and 700, the maximum activity was below 50 events per week, even in case of the high deformation velocity of above $20 \mathrm{~mm} /$ day (Fig. 4).

Due to the qualitative character of the observed relation between the seismic activity and the benchmark displacement velocity, the quantitative confirmation of the presented results requires further investigation and measurements performed with higher frequency and in various geological and mining conditions.

Acknowledgments I express my gratitude to prof. Wacław M. Zuberek for the advice, discussion and cooperation. This outcome has been achieved with the support of the Ministry of Sciences and High Education of Poland, Project No N N525 39839. The research has been carried out in the Faculty of Earth Sciences of the University of Silesia in Katowice. Additional calculations and analyses have been carried out in the Faculty of Civil Engineering and Architecture of Opole University of Technology.

Open Access This article is distributed under the terms of the Creative Commons Attribution 4.0 International License (http://creativecommons.org/licenses/by/4.0/), which permits unrestricted use, distribution, and reproduction in any medium, provided you give appropriate credit to the original author(s) and the source, provide a link to the Creative Commons license, and indicate if changes were made.

\section{References}

Bańka P (2000) Dependence of induced seismicity on the change of rock mass deformation state defined in the function of time. Acta Mont IRSM AS CR Ser A 16(118):7-16

Bian H, Zhang S, Zhang Q, Zheng N (2014) Minitoring large area mining subsidence by GNSS based on IGN stations. Trans Nonferrous Met Soc China 24:514-519. doi:10.1016/S1003-6326(14)63090-9

Bogusz M, Mendecki M (2011) Seismic and geodetic observation of subsidence through development over a longwall face in the coal bed under extraction. In: Idziak AF, Dubiel R (eds) Geophysics in mining and environmental protection, geoplanet: earth and planetary sciences, vol 2. Springer, Berlin, pp 71-79. doi:10.1007/978-3-642-19097-1

Cai Y, Verdel T, Deck O (2014) On the topography influence on subsidence due to horizontal underground mining using the influence function method. Comput Geotech 61:328-340. doi:10.1016/j.compgeo. 2014.06.003

Dębski W (2010) Probabilistic inverse theory. Adv Geophys 52:1-102. doi:10.1016/S0065-2687(10)52001-6

Deck O, Anirudh H (2010) Numerical study of the soil-structure interaction within mining subsidence areas. Comput Geotech 37:802-816. doi:10.1016/j.compgeo.2010.07.001

Doležalová H, Kajzar V, Soucek K, Stas L (2009) Evaluation of mining subsidence using GPS data. Acta Geodynamica et Geomaterialia 3(155):359-367

Gibowicz SJ, Kijko A (1994) An introduction to mining seismology. Academic Press, San Diego

Knothe S (1959) Observations of surface movements and their theoretical interpretation. Colliery Eng $36: 24-29$

Knothe S (1984) Forecasting of the influence of underground mining [in Polish]. Śląsk, Katowice

Knothe S (2005) Asymmetric function of distribution of mining exploitation influences in the medium with changing properties. Arch Min Sci 50:401-415

Kochmański T (1980) Theory of Tadeusz Kochmanski [in Polish]. In: Borecki M (ed) Ochrona powierzchni przed szkodami górniczymi. Śląsk, Katowice, pp 213-239

Kowalski A (2001) Surface subsidence and rate of its increments based on measurements and theory. Arch Min Sci 46(4):391-406

Kowalski A (2004) Prediction of transient deformations of the surface including the rheological models in the Szkody 4.1 system. Arch Min Sci 41(1):3-28

Kowalski A (2007) Transient mining surface deformations in the prediction accuracy aspect [in Polish]. Główny Instytut Górnictwa, Katowice 
Saeidi A, Deck O, Verdel T (2009) Development of building vulnerability functions in subsidence regions from empirical methods. Eng Struct 31:2275-2286. doi:10.1016/j.engstruct.2009.04.10

Sokoła-Szewioła V (2009) Method of prediction the probability of a strong tremor on the basis of observed changes of mining ground subsidences. Arch Min Sci 54(4):725-737

Sroka A (2009) Influence of time based parameters of longwall panel exploitation on objects inside rock mass and on the surface. Arch Min Sci 54(4):819-826

Wiemer S, Wyss M (2002) Mapping spatial variability of the frequency-magnitude distribution of earthquakes. In: Dmowska R, Saltzman B (eds) Advances in Geophysics, vol 45. Elsevier, Amsterdam, pp 259-302. doi:10.1016/S0065-2687(02)80007-3 